\title{
Understanding the Neutron Star Population with the SKA
}

\author{
Thomas M. Tauris ${ }^{1,2}$, Victoria M. Kaspi ${ }^{* 3}$, Rene P. Breton ${ }^{4}$, Adam T. Deller $^{5}$, \\ Evan F. Keane ${ }^{6,7}$, Michael Kramer ${ }^{2}$, Duncan R. Lorimer ${ }^{8}$, Maura A. McLaughlin ${ }^{8}$, \\ Andrea Possenti ${ }^{9}$, Paul S. Ray ${ }^{10}$, Ben W. Stappers ${ }^{11}$, Patrick Weltevrede ${ }^{11}$ \\ ${ }^{1}$ Argelander-Institute for Astronomy, University of Bonn, Germany; ${ }^{2}$ MPIfR, Germany; ${ }^{3}$ McGill \\ University, Canada; ${ }^{4}$ University of Southampton, UK; ${ }^{5}$ ASTRON, The Netherlands; ${ }^{6}$ Swinburne \\ University of Technology, Australia; ${ }^{7}$ ARC Centre of Excellence for All-Sky Astrophysics \\ (CAASTRO); ${ }^{8}$ West Virginia University, USA; ${ }^{9}$ INAF-Osservatorio Astronomico di Cagliari, \\ Italy; ${ }^{10}$ Naval Research Laboratory, USA; ${ }^{11}$ University of Manchester, UK \\ E-mail: tauriseastro.uni-bonn.de vkaspiephysics.mcgill.ca
}

Since their discovery in the late 1960's the population of known neutron stars has grown to $\sim 2500$. This sample has yielded many surprises and demonstrated that the observational properties of neutron stars are remarkably diverse. The surveys that will be performed with the SKA will produce a further tenfold increase in the number of Galactic neutron stars known. Moreover, the SKA's broad spectral coverage, sub-arraying and multi-beaming capabilities will allow us to characterise these sources with unprecedented efficiency, in turn enabling a giant leap in the understanding of their properties. We review the neutron star population and outline strategies for studying each of the growing number of diverse classes that are populating the "neutron star zoo". Questions that will be addressed by the much larger statistical samples and vastly improved timing efficiency provided by SKA include: (i) the spin period and spin-down rate distributions (and thus magnetic fields) at birth, and the associated information about the supernovae wherein they are formed; (ii) the radio pulsar-magnetar connection; (iii) the link between normal radio pulsars, intermittent pulsars and rotating radio transients; (iv) the slowest possible spin period for a radio pulsar (revealing the conditions at the pulsar death-line); (v) proper motions of pulsars (revealing supernova kick physics); (vi) the mass distribution of neutron stars; (vii) the fastest possible spin period for a recycled pulsar (constraining magnetosphere-accretion disc interactions, gravitational wave radiation and the equation-of-state); (viii) the origin of high eccentricity millisecond pulsars; (ix) the formation channels for recently identified triple systems; and finally (x) how isolated millisecond pulsars are formed. We can also expect that the first phase of the SKA (SKA1), and in particular the full SKA (SKA2), will break new ground unveiling exotic and heretofore unknown systems that will challenge our current knowledge and theories, thus fostering the development of new research areas. Some possibilities for future landmark discoveries representing significant milestones in the astrophysics of compact objects include: (i) sub-millisecond pulsars; (ii) neutron stars born as millisecond pulsars; (iii) neutron stars with masses below 1.1 or above $2.5 M_{\odot}$; (iv) neutron star-black hole binaries; and (v) a triple system containing a pair of neutron stars.

Advancing Astrophysics with the Square Kilometre Array

June 8-13, 2014

Giardini Naxos, Italy

\footnotetext{
* Speaker.
} 


\section{Introduction}

The recent era of multi-wavelength observations has revealed a greater variety of possibly distinct observational classes of neutron stars (NSs) than ever before. In addition to isolated NSs, these compact objects are found in binaries and even triple stellar systems which demands theoretical research on their origin and evolution, besides enabling precise mass measurements. With emission spanning the entire electromagnetic spectrum and some NSs showing strange transient behaviour and even dramatic high-energy outbursts, such incredible range and diversity was not only unpredicted, but in many ways astonishing given the perhaps naively simple nature of the NS - the last stellar bastion before the total collapse to a black hole. But why do NSs exhibit so much 'hair'? Even within the field there is confusion about the sheer number of different NS class nomenclature. For a recent review on the members of the NS zoo and the possible unification of the various flavours, we refer to Kaspi (2010) and references therein. An overview of the formation and evolution of NSs in binaries is given, for example, in Bhattacharya \& van den Heuvel (1991); Tauris \& van den Heuvel (2006).

In Figure 1 we have plotted all currently known radio pulsars with measured values of spin period $(P)$ and its time derivative $(\dot{P})$. The differences in $P$ and $\dot{P}$ imply fundamentally different magnetic field strengths and ages for each sub-group of the population. Treating the pulsar as a

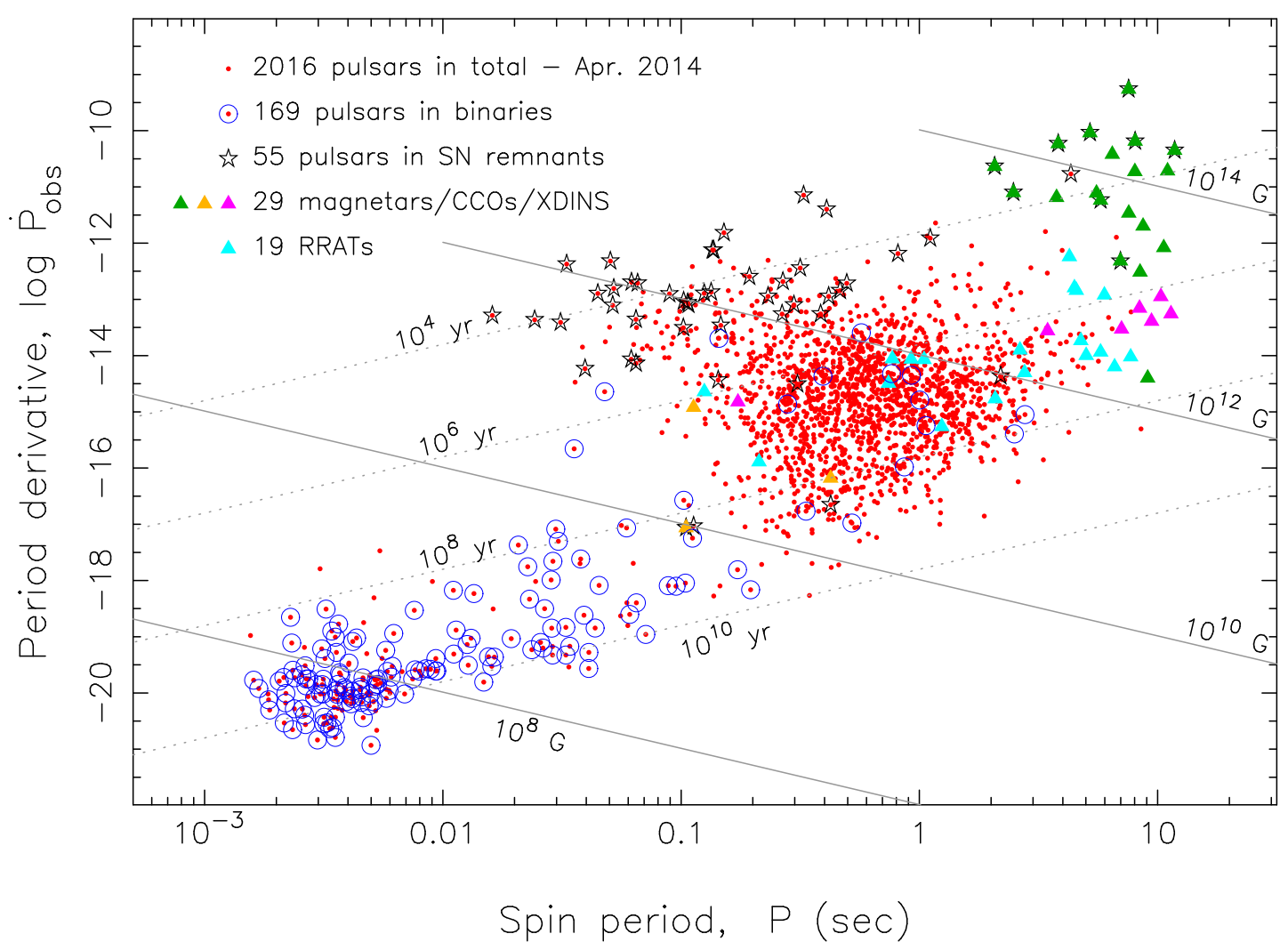

Figure 1: All currently known pulsars with measured $P$ and $\dot{P}$. Data from the ATNF Pulsar Catalogue (Manchester et al. 2005) - April 2014. Lines of constant characteristic age and constant B-field are marked. 
rotating magnetic dipole, one may show (e.g. Lorimer \& Kramer 2004) that the surface magnetic field strength $B \propto(P \dot{P})^{1 / 2}$ and define a characteristic age $\tau_{\mathrm{c}}=P /(2 \dot{P})$. The classic radio pulsars (red dots) are concentrated in the region with $P \simeq 0.2-2 \mathrm{sec}$ and $\dot{P} \simeq 10^{-16}-10^{-13}$. They have magnetic fields of the order $B \simeq 10^{10}-10^{13} \mathrm{G}$ and a lifetime as a radio source of a few $10^{7} \mathrm{yr}$. The population plotted in blue circles are binary pulsars and clearly indicate a connection to the rapidly spinning millisecond pulsars. The pulsars marked with stars indicate young pulsars observed inside, or near, their gaseous supernova ( $\mathrm{SN})$ ejecta remnants. Then there are the "drama queens" of the NS population: the magnetars which undergo high-energy bursts and are powered by their huge magnetic energy reservoirs. Also plotted are the mysterious rotating radio transients (RRATs), the isolated X-ray dim NSs (XDINS) and the compact central objects (CCOs). In addition, we briefly discuss other exotic radio pulsars such as the intermittent pulsars, the black widows and redbacks, and pulsars in triple systems.

A main challenge of the past decade was - and continues to be - to find a way to unify this variety into a coherent physical picture. The NS zoo raises essential questions like: What determines whether a NS will be born with, for example, magnetar-like properties or as a Crablike pulsar? What are the branching ratios for the various varieties, and, given estimates of their lifetimes, how many of each are there in the Galaxy? Can individual NSs evolve from one species to another (and why)? How does a NS interact with a companion star during and near the end of mass transfer (recycling)? And what limits its final spin period? Ultimately such questions are fundamental to understanding the fate of massive stars, close binary evolution and the nature of core-collapse $\mathrm{SNe}$, while simultaneously relating to a wider variety of interesting fundamental physics and astrophysics questions ranging from the physics of matter in ultra-high magnetic fields, to the equation-of-state of ultra-dense matter and details of accretion processes.

In this chapter we address the many faces of NSs with a strong emphasis on radio pulsars and how the SKA will push this research area further to the forefront of modern astrophysics. The structure of the chapter follows the NS population from its very youngest sources, that still bear the imprint of their birth conditions, to the oldest recycled millisecond pulsars, resulting from interactions with a companion star on a Gyr timescale. The bulk of the NSs are somewhere in the middle of their evolutionary journey, so that ultimately detailed population synthesis studies of the full bounty offered by future SKA searches will be key to a complete picture. Whereas the chapter on "A Cosmic Census of Radio Pulsars" (Keane et al. 2015) addresses more technical aspects and emphasizes what sources might be found, this chapter has more specific focus on the consequences of these discoveries and how they would relate to the general population of neutron stars and our understanding thereof.

\section{Magnetars}

Without doubt the most dramatic and radiatively unpredictable of NSs are the so-called "magnetars," believed to be very young and the most highly magnetized of the population. These objects, of which 26 are currently known (Olausen \& Kaspi 2014) ${ }^{1}$, have as their hallmark emission bright occasional super-Eddington bursts of X-rays and soft gamma rays. The energy source for these

\footnotetext{
${ }^{1}$ See online magnetar catalogue at http://www.physics.mcgill.ca/ pulsar/magnetar/main.html
} 
bursts, as well as for the luminous X-ray pulsations observed from many in this class of objects, is believed to be the decay of an intense internal magnetic field, which causes stresses and fractures of the stellar crust (Thompson \& Duncan 1995, 1996). These fields are inferred from a variety of different arguments (see, Thompson \& Duncan 1996) but arguably most commonly from the spin period and period derivative measured from the $\mathrm{X}$-ray pulsations via the conventional dipole spin-down formula: $B=3.2 \times 10^{19} \sqrt{P \dot{P}} \mathrm{G}$, often yielding dipolar B-fields $>10^{14} \mathrm{G}$. Outstanding questions regarding magnetars include the origin of such high magnetic fields (Damour \& Taylor 1992), what fraction of the NS population is born as magnetars (Keane \& Kramer 2008), and whether there is an evolutionary relationship between magnetars and other NSs, such as highmagnetic-field radio pulsars (see Section 3 below) or isolated NSs (Turolla 2009). Recently, Klus et al. (2014) have suggested that magnetars may populate a group of high-mass X-ray binaries observed in the Small Magellanic Cloud.

Of the 26 known magnetars, only four have been radio-detected thus far (Camilo et al. 2006, 2007a; Levin et al. 2010; Eatough et al. 2013) in spite of sensitive searches of many others (Burgay et al. 2006; Crawford et al. 2007; Lazarus et al. 2012). The four solidly detected magnetars show interesting radio properties. The first radio-detected magnetar, XTE J1810-197 (Camilo et al. 2006), became a bright radio pulsar only after its 2003 X-ray outburst, and showed a remarkably flat radio spectrum, at some point being the brightest known radio pulsar above $20 \mathrm{GHz}$. Extreme variability as well as a very high degree $(\sim 100 \%)$ of linear polarization were also observed (Kramer et al. 2007; Camilo et al. 2007b) as well as dramatic pulse profile changes (Camilo et al. 2007c). Meanwhile, the second-detected radio magnetar, 1E 1547.0-5408 (Camilo et al. 2007a), has shown properties quite similar to those of XTE J1810-197. A third radio-loud magnetar, PSR J1622-4950, was discovered (Levin et al. 2010) in apparent X-ray quiescence during a standard survey for radio pulsars, hence making it the first radio-discovered magnetar. It too was characterized by extreme variability, with epochs during which it was undetectable, and a flat or even inverted radio spectrum. Subsequent X-ray observations suggest that it is likely recovering from an earlier X-ray outburst (Anderson et al. 2012), further suggesting that magnetar radio emission is associated with post-outburst events.

Very recently, a fourth radio magnetar, SGR J1745-29, was discovered via its X-ray emission, only $3^{\prime \prime}$ from the Galactic Centre (Mori et al. 2013). The subsequent radio detection (Eatough et al. 2013; Shannon \& Johnston 2013) showed again high linear polarization, and the largest dispersion and rotation measures for any known pulsar. In contrast to the other three radio-detected magnetars, this source appears to be far less radio variable, even as the X-ray emission fades (Kaspi et al. 2014; Lynch \& Kaspi 2015). Interestingly, the high Faraday rotation as inferred from radio observations argues for a dynamically significant magnetic field near the central super-massive black hole, relevant to the physics of the accretion flow (Eatough et al. 2013). Importantly, a multiwavelength pulse profile study (Spitler et al. 2014) shows the pulse is scatter-broadened by an order of magnitude less than was predicted by the in-standard-use Cordes \& Lazio (2002) model, a conclusion supported by radio angular broadening measurements (Bower et al. 2014). This is significant for potential SKA pulsar searches of the Galactic Center region (see, Eatough et al. 2015; Keane et al. 2015), as it implies pulsars may be easier to find there than has been previously thought (Chennamangalam \& Lorimer 2014), particularly at high radio frequencies such as SKA1MID band 5, even in spite of the very few detections thus far in relatively high-frequency surveys 
(Bates et al. 2011). The radio detections of pulsars within the central parsec of the Galaxy could allow unique dynamical tests that could constrain properties of the central super-massive black hole as well as test theories of gravity (Pfahl \& Loeb 2004; Liu et al. 2012; Eatough et al. 2015).

\subsection{The contribution of SKA}

The SKA will be of great use for the radio study of magnetars. Current upper limits on radio observed, but yet undetected, sources are in the range of 0.01-0.04 mJy for periodic emission and 0.1-10 mJy for single pulses, depending on pulse width (e.g. Lazarus et al. 2012). SKA sensitivities are up to an order of magnitude better than was previously possible, thereby greatly increasing the available phase space for detection at a wide range of frequencies. The detection and/or improved upper limits for additional sources will help determine if the bulk are indeed radio quiet, as might be expected in some models of pulsar emission (Baring \& Harding 2001) or due to small beaming angles (Lazarus et al. 2012). The SKA detection of radio emission from a magnetar which previously appeared to have faded beyond observability in the radio band could demonstrate persistent low-level radio emission. A comparison of the radio properties of such a source in radio quiescence with those when it was in outburst may be further constraining of models. Differences in polarization profiles between these two states could prove to be highly effective diagnostics of geometric differences expected in twisted magnetosphere models (Thompson et al. 2002; Beloborodov \& Thompson 2007). Regular monitoring of magnetars with SKA-type sensitivities could help determine whether their hallmark bursts are present in the radio band as has been suggested in some burst models (Lyutikov 2002). Furthermore, some authors have suggested fundamental physics experiments with magnetar radio emission, such as axion detection using photon beam splitting (Guendelman \& Chelouche 2011), which may be facilitated with more sources detected.

\section{High B-Field Radio Pulsars}

One question regarding the NS population is what distinguishes magnetars from otherwise apparently conventional radio pulsars which appear, from their $P$ and $\dot{P}$, to have magnetar-strength B-fields. Indeed currently there are 9 known, otherwise conventional radio pulsars, having spininferred surface dipolar magnetic field strengths above $4.4 \times 10^{13} \mathrm{G}$ (a somewhat arbitrary limit derived from the so-called "quantum critical field" $B=m^{2} c^{2} / \hbar e$ ), with the highest known being $B=9.4 \times 10^{13} \mathrm{G}$ for PSR J1847-0130. Such fields clearly overlap, and in some cases exceed, those of magnetars, for which the smallest spin-inferred dipolar magnetic field is a paltry $B=$ $6.1 \times 10^{12} \mathrm{G}$ (Rea et al. 2013). Therefore, there is reason to expect potential magnetar-like behavior in high-B radio pulsars. Indeed, PSR J1846-0258 in the SN remnant Kes 73 has shown magnetarlike phenomenology (Gavriil et al. 2008) in the form of a short-lived X-ray outburst simultaneous with a large rotation glitch. Deep X-ray observations of high-B radio pulsars using telescopes like Chandra and XMM-Newton have also shown strong evidence for enhanced thermal X-ray emission from high-B radio pulsars relative to that seen in lower-B radio pulsars of comparable age (Kaspi \& McLaughlin 2005; Zhu et al. 2011; Olausen et al. 2013). This could be understood in terms of magneto-thermal evolution in NSs, in which B-fields decay internally, releasing energy and keeping the NS hot (Aguilera et al. 2008; Perna et al. 2013). 


\subsection{The contribution of SKA}

The discovery of new high-B radio pulsars in surveys with the SKA has the potential to make important progress in this field, primarily because of the paucity of high-B pulsars currently known. With new examples, together with follow-up X-ray observations, particularly with future highthroughput X-ray missions such as ATHENA ${ }^{2}$, additional data points can be added on NS cooling curves (Olausen et al. 2013). Given the expectations for the numbers of pulsars to be discovered with SKA, and assuming the current ratio of high-B radio pulsars to the rest of the population ( $\sim 0.4 \%$ ) continues to hold, SKA should discover 50-100 new high-B sources for X-ray follow-up.

Moreover, regular monitoring of these high-B radio pulsars for timing purposes is likely to reveal many spin glitches, particularly given how ubiquitous and dramatic such events are in magnetars (Dib et al. 2008; Dib \& Kaspi 2014): spin-up and -down glitches have been observed and are generally accompanied by flux enhancements as well as pulse profile changes and bursts. The timely detection of a glitch in a high-B radio pulsar could yield an opportunity for rapid X-ray follow-up observations to search for an X-ray outburst. Indeed, there already exist three examples of high-B radio pulsars exhibiting interesting pulse variability at glitch epochs (Gavriil et al. 2008; Weltevrede et al. 2011; Keith et al. 2013). The discovery of new, young high-B radio pulsars could permit the measurement of more braking indices. The young radio pulsar PSR J1734-3333 has $B=5 \times 10^{13} \mathrm{G}$, and a braking index of only $n=0.9 \pm 0.2$ (Espinoza et al. 2011), the lowest yet measured for any radio pulsar and well below the canonical $n=3$ expected for magnetic dipole radiation in a vacuum. Such a low braking index can be interpreted as resulting from magnetic-field growth, suggesting that magnetars could be descendants of high-B radio pulsars.

\section{Central Compact Objects and Neutron Stars in Supernova Remnants}

Another group of NSs are the central compact objects (CCOs; see e.g. Pavlov et al. 2002). These are isolated, non-variable point sources associated with supernova remnants (SNRs), seen in thermal X-rays. So far they are without optical or radio counterparts (although the latter are single-dish limits). CCOs have low X-ray luminosities $\left(\sim 10^{33} \mathrm{erg} \mathrm{s}^{-1}\right)$ and do not have associated pulsar wind nebulae, suggesting that these stars are NSs which may not yet be active as pulsars. There are currently eight confirmed CCO sources plus three candidates ${ }^{3}$ (Weisskopf et al. 2006; de Luca 2008; Gotthelf et al. 2013) and multi-wavelength observing campaigns have been undertaken to search for more sources in nearby SNRs (Kaplan et al. 2006). For three of the CCOs, the spin characteristics $(P$ and $\dot{P}$ ) are known (see Figure 1) from X-ray timing observations and indicate surprisingly low magnetic fields ( $\sim 10^{10} \mathrm{G}$, now in two cases, e.g. Gotthelf et al. (2013)).

Despite the small number of known CCOs it is possible to estimate a minimum Galactic birth rate of these sources using the ages estimated from the SNR expansion times. This yields $\beta_{\mathrm{CCO}} \sim 0.5$ century $^{-1}$ (Gaensler et al. 2000). CCOs are thus a significant contributor to the socalled "NS birthrate problem" (Keane \& Kramer 2008): the problem that arises if one assumes all of the various NS manifestations discussed in this chapter are evolutionarily independent. Their cumulative birthrate does not tally with the estimated core-collapse SN rate for the Galaxy and so the

\footnotetext{
${ }^{2}$ http://www.the-athena-x-ray-observatory.eu/

${ }^{3}$ Some authors also define 1E 161348-5055, the variable X-ray source associated with the RCW 103 SNR as a CCO. However here we follow the definition of Halpern \& Gotthelf (2010) and take CCOs as having steady X-ray flux.
} 
various groups must evolve into one another along as yet uncertain evolutionary sequences. Moreover, the spin-inferred magnetic fields of CCOs are so small that their expected lifetimes above the death-line in the $P-\dot{P}$ diagram are tens to hundreds of millions of years; this conflicts strongly with the apparent paucity of sources in this region of the $P-\dot{P}$ diagram, if the typical CCO is a radio emitter. CCOs are thus a very important part of the puzzle on NS evolutionary pathways.

\subsection{The contribution of SKA}

A deep investigation with both SKA1 and SKA2 could determine once and for all whether CCOs are radio emitters. In addition to the CCOs, there are currently 55 pulsars with SNR associations. Figure 1 shows that these sources are predominantly "young" with $\tau_{\mathrm{c}}=10^{4-5} \mathrm{yr}$, consistent with the short lifetimes of visible SNRs. The "older" sources with SNR associations are either chance superpositions (Bogdanov et al. 2014) or are in fact young, i.e. having been born with a slow birth period causing their characteristic age to be much larger than their true age. The SKA will enable unprecedented deep searches of all of the Galactic SNRs visible in the SKA-sky and will firmly detect all of those showing even minute levels of radio pulsar emission. Timing these pulsars with the SKA will allow measurement of their spin characteristics and thence enable us to "rewind" the spin history of these stars to determine a more realistic birth period distribution for pulsars, a notoriously difficult aspect of pulsar evolution to model (Faucher-Giguère \& Kaspi 2006; Noutsos et al. 2013).

\section{Rotating Radio Transients}

Rotating radio transients (RRATs) are a class of pulsars from which pulses are only sporadically detectable (McLaughlin et al. 2006). The appropriate definition of this class of objects, and indeed whether a definition is even necessary, has been debated (Keane \& McLaughlin 2011). Here, we define RRATs as NSs which were originally detectable only through their single pulses and not through a periodicity search (though we exclude the first pulsars discovered before the regular application of periodicity searches). Using this definition, there are roughly 100 known RRATs ${ }^{4}$. Their periods range from $0.125 \mathrm{~s}$ to $7.7 \mathrm{~s}$, with a mean of $2.6 \mathrm{~s}$. A fraction $(\sim 20 \%)$ of the RRATs do not yet have determined periods due to a small number of detected pulses. These periods show some evidence of being longer than those of other pulsars. It is unclear if longer-period pulsars become sporadic emitters, or if this is a selection effect due to the smaller number of pulses in an observation - and hence, for longer period pulsars, higher likelihood of being more detectable in a single-pulse search.

Measuring period derivatives for RRATs often requires much more observing time than for normal pulsars due to their sporadic emission. Therefore, only $\sim 25 \%$ of the RRATs have measured period derivatives (cf. Figure 1). These do not appear to be significantly different than the period derivatives of other pulsars, but due to the longer periods, the inferred surface dipole magnetic fields of the RRATs are possibly higher than for other pulsars, with a mean of $3 \times 10^{12} \mathrm{G}$.

When the discovery of the RRATs was first announced (McLaughlin et al. 2006), it was believed that they were a new class of radio-emitting NSs. Under this assumption, their sporadic

\footnotetext{
${ }^{4}$ See the online catalogue of RRATs at http://astro.phys.wvu.edu/rratalog
} 
properties imply that for each detected RRAT, there are many more undetected objects, leading one to infer a very large population of these sources, and hence NSs, in the Galaxy (McLaughlin et al. 2006). This issue was further explored and Keane et al. (2010) found that, assuming that the RRATs form a population distinct to other NSs, the total inferred number of Galactic NSs is discrepant with Galactic SN rate estimates. One solution to this problem is if RRATs are a part of the same population as other pulsars.

This assumption seems reasonable, given the similarity between the properties of RRATs and other pulsars, and the apparent continuum in intermittency bridging these two (perhaps not distinct) populations. Studies of the single pulses of RRATs indicate that their brightness temperatures are no greater than the single-pulse brightness temperatures for other pulsars. In addition, the pulse shapes and widths of RRAT pulses are similar to those of other long-period pulsars. Furthermore, some RRATs were initially detectable only through their single pulses, but with observations at a lower frequency or with a more sensitive telescope, they are detectable through periodicity searches like normal pulsars. It is therefore plausible to assume that RRATs are simply pulsars which sit on the tail end of a continuous intermittency spectrum and are not part of any special population.

\section{Intermittent Pulsars}

While there has been progress in the theoretical understanding of the magnetospheric processes in pulsars (e.g. Contopoulos et al. 1999; Spitkovsky 2006), some of the most important experimental insight comes from the study of a class of NSs called intermittent pulsars. The archetype of this relatively recently discovered class is radio pulsar B1931+24 (Kramer et al. 2006a; Young et al. 2013), which is only active for a few days between periods of roughly a month during which the pulsar is not detectable. The fact that pulsars are able to switch on and off is something that has been known for a long time and is often observed at much shorter timescales, in which case it is known as nulling (e.g. Backer 1970; Wang et al. 2007). The exciting result for the intermittent pulsars is that these sudden switches are accompanied by changes in the spin-down rate of the NS rotation, suggestive of significant changes in the torque generated by magnetospheric currents. For PSR B1931+24 the spin-down was shown to be 50\% larger when the radio emission of the pulsar is in the on-state compared to the off-state (Kramer et al. 2006a), explaining at the same time the seemingly noisy timing behaviour for this source (see Figure 2).

Besides PSR B1931+24, there are only two other intermittent pulsars known which change their spin-down rate. For PSR J1832+0029 (Lorimer et al. 2012) the spin-down rate changes by a factor of 1.8, while for PSR J1841-0500 (Camilo et al. 2012) the spin-down rate changes by a factor of 2.5. All three intermittent pulsars appear to be part of the bulk of the pulsars in the $P-\dot{P}$ diagram. At first, it may seem somewhat surprising that radio emission is linked to the spin-down rate of pulsars. The amount of energy in the radio emission is tiny in comparison to the total loss rate of the rotational energy of the NS (i.e. $\dot{E}$ ), so one could expect the radio emission to be an indicator of higher order effects rather than fundamental processes within the pulsar magnetosphere. Nevertheless, the radio emission appears to be a tracer of powerful currents in the magnetosphere.

The behavior of intermittent pulsars can be explained by a relatively simple two-state magnetospheric model (Kramer et al. 2006a; Li et al. 2012). In one of the states the open field line region is filled with a co-rotating plasma which as a consequence should have the Goldreich-Julian den- 


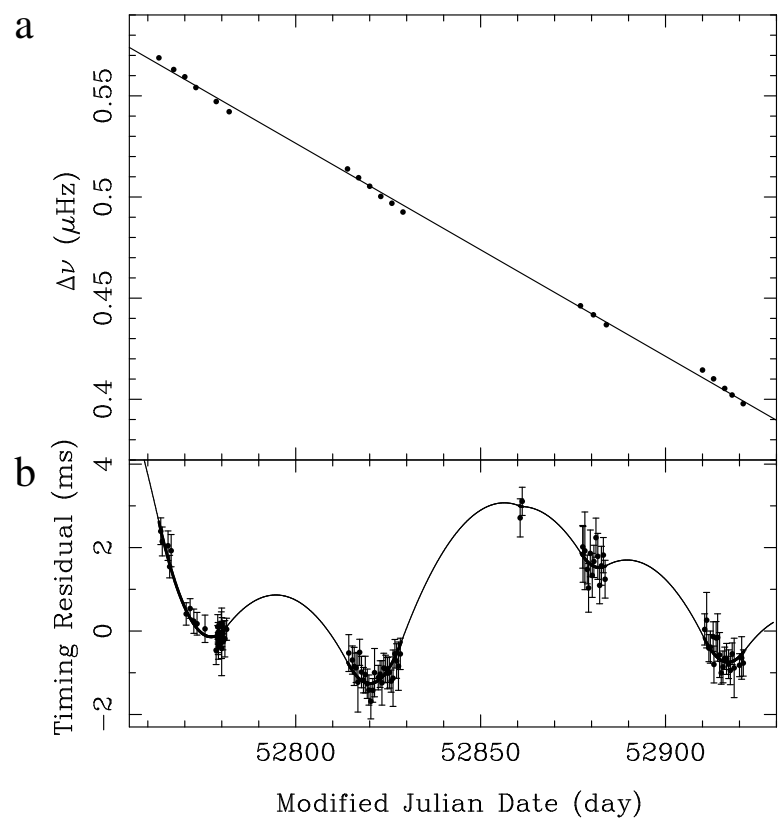

Figure 2: Changes in the spin-rate of PSR B1931+24 (upper panel) when 'on' (radio bright). The rotational frequency is clearly decreasing faster (i.e. the gradient is steeper) in the 'on'-state compared to the average spin-down rate (indicated by the solid line), implying that the pulsar is spinning down slower in the 'off' (radio quiet) state. Taking the switch between two different slow-down rates into account, the apparent noisy timing behaviour can be explained perfectly (lower panel). Figure from Kramer et al. (2006a).

sity (Goldreich \& Julian 1969). This magnetospheric state can in good approximation be described by a force-free magnetospheric solution (Spitkovsky 2006). This plasma is generated by the same mechanism which produces the radio emission observed during the on-state. When this mechanism switches off both the radio emission and plasma production ceases, resulting in the open field line region to get depleted from plasma. This vacuum state will have a different spin-down rate by a factor which depends on the magnetic inclination angle (Li et al. 2012), which can explain the range (1.8-2.5) in observed factors by which the spin-down rate is changing.

An alternative model has been suggested (Timokhin 2010) in which the switching between stable magnetospheric states causes different geometries or/and different distributions of currents which can explain the persistent "nulling" during the off-states. Furthermore, it has become clear that there are more subtle ways in which the radio emission and spin-down rate are linked. There are a number of objects which show pulse profile changes concurrent with changes in the spin-down rate (Lyne et al. 2010). This has led to the suggestion that all the so-called timing-noise (deviations from a simple spin-down model) could be ascribed to switches between magnetospheric states. These more subtle changes in the emission result in more subtle changes in spin-down, which can be less than $1 \%$. One should note, however, that most of these state changes (e.g. the so-called "moding") happens on time-scales of minutes to hours and are therefore too fast to measure (and, hence, separate) correlated changes in spin-down rate (Young et al. 2012). 


\subsection{The contribution of SKA}

The SKA can help in several ways to make progress in our understanding of the pulsar magnetosphere using intermittent pulsars (see also Karastergiou et al. 2015). Firstly, we will progress by discovering more intermittent pulsars. This will lead to additional measurements of the changes in the spin-down rate between the on- and the off-state for many more sources, thus understanding better the magnetospheric state changes. For this it will be important, however, that repeated passes are performed over given search areas. The time between these passes should vary from hours and days to weeks and months, similar to the range of time scales observed for both intermittent and nulling pulsars (as well as RRATs, see Section 5). Indeed, an important question which the SKA will help to address is what the difference (if any) is between intermittent pulsars and nulling pulsars, as the main difference appears to be the duration of the off-state.

It is important to note that the large sensitivity of the SKA will not necessarily mean that we can detect much smaller changes in the spin-down rate or changes which are shorter in duration. However, the high sensitivity of the SKA can put better (important) limits on the presence of radio emission during the off-state. It is easy to confuse nulls with very weak emission, as has been shown for instance for PSR B0826-34 (Esamdin et al. 2005). The presence of very weak emission during the off-state of intermittent pulsars would show that there are at least some currents present in the pulsar magnetosphere at all times. Additionally, the multi-beaming capability of the SKA will allow a dense monitoring of the known intermittent (and other) pulsars. The much larger possible cadence will allow us to determine the exact switch times between the states more accurately. This is important for two reasons: i) we can probe the plasma conditions much better by constraining the currents present in the magnetospheres and correlate the results to other properties of pulsars, and ii) we can potentially use the information to correct for timing noise in non-recycled pulsars, hence, opening up the possibility to use them also for high precision experiments such as gravitational wave detection (Lyne et al. 2010).

\section{Isolated Pulsar Evolution on the $P-\dot{P}$ Diagram}

A common approach to understand the distribution of pulsars in the $P-\dot{P}$ diagram (Figure 1) is to take a prescription for the spin evolution of NSs (often a simple magnetic dipole model) and evolve pulsars forward from some birth distributions in $P$ and $B$ in time to find the present-day distribution. Ideally, after accounting for observational selection effects (Emmering \& Chevalier 1989), this distribution would be statistically indistinguishable from the one that is observed. The major challenge facing all these studies is to decouple various competing and covariant effects that shape this distribution. Assuming that radiation beaming (Tauris \& Manchester 1998) and the selection effect modelling process (Faucher-Giguère \& Kaspi 2006) are reasonably well understood, the main factors involved are discussed in turn below. A less model dependent approach is to compute the flux or "current" of pulsars through the $P-\dot{P}$ diagram by taking into account detection biases (Phinney \& Blandford 1981; Vivekanand \& Narayan 1981; Narayan 1987). Recently, Lee et al. (2012) have attempted to categorize sub-populations within the distribution using Gaussian mixture models. The main factors for shaping the pulsar distribution are: 
- Neutron star birth parameters. While the overall consensus on the distribution of B-fields seems to favour a single-component log-normal distribution, much debate has taken place over the form of the period distribution. The view put forward by early studies (e.g. Lyne et al. 1985) which favoured a model in which all pulsars were born spinning rapidly was challenged by the idea of an "injection" of pulsars into the population with birth periods of around $0.5 \mathrm{~s}$ (Vivekanand \& Narayan 1981; Narayan 1987; Narayan \& Ostriker 1990). Current studies (Faucher-Giguère \& Kaspi 2006; Ridley \& Lorimer 2010) favour a broad normal distribution with a mean of $0.3 \mathrm{~s}$ and standard deviation of $0.5 \mathrm{~s}$. Independent constraints on the initial period distribution from pulsar-SN remnant pairs (Popov \& Turolla 2012) suggest a mean and standard deviation of $0.1 \mathrm{~s}$.

- Magnetic field strength evolution. Solving the differential equation $B \propto(P \dot{P})^{1 / 2}$ to obtain the evolution of $P$ and $\dot{P}$ with time in the magnetic dipole model is most readily achieved assuming that $B$ is constant. Such models provide good matches to the $P$ - $\dot{P}$ distribution (Bhattacharya et al. 1992; Faucher-Giguère \& Kaspi 2006). However, models which invoke a very modestly decaying magnetic field (Popov et al. 2010) are also consistent with the data. Earlier theoretical work (Tauris \& Konar 2001) also reached similar conclusions.

- Magnetic inclination evolution. Within the frame of a rotating magnetic dipole in a vacuum, the torque acting on the spinning NS is proportional to $B^{2} \sin ^{2} \alpha$, where $\alpha$ is the angle between the spin and magnetic axes. This relationship implies that magnetic field decay mentioned in the previous item can also be mimicked by a decay in the inclination angle, $\alpha$. Several independent studies (Tauris \& Manchester 1998; Weltevrede \& Johnston 2008; Young et al. 2010) conclude that $\alpha$ decays with time. An outstanding issue (Ridley \& Lorimer 2010 ) is how this decay translates to a change in the braking torque on the pulsar. While Tauris \& Konar (2001) suggest that a decay in the braking torque is required by the $P-\dot{P}$ distribution, as Ridley \& Lorimer (2010) demonstrate, Monte Carlo simulations assuming magnetic alignment cannot satisfactorily explain the observed sample.

- Non-standard magnetic dipole evolution. One possible solution to the alignment issue discussed above is to evolve the pulse period according to a different spin-down law. A revised model has been proposed (Contopoulos \& Spitkovsky 2006) with different evolutionary tracks which allow for a constant magnetic field and alignment. Detailed Monte Carlo models (Ridley \& Lorimer 2010) have, however, so far not been able to reproduce the $P-\dot{P}$ diagram using this framework.

- Luminosity "law" and evolution. In the absence of a well developed theoretical framework for understanding the radio emission of pulsars, the luminosity $L$ is often calculated from a power-law expression of the form $L \propto P^{\alpha} \dot{P}^{\beta}$. This approach has been adopted by numerous studies (e.g. Lyne et al. 1985; Stollman 1987; Narayan \& Ostriker 1990; Faucher-Giguère \& Kaspi 2006), with quite different values for the exponents $\alpha$ and $\beta$. It is noteworthy (Lorimer et al. 1993) that the adopted values for $\alpha$ and $\beta$ significantly impact the conclusions regarding population parameters. For example, combinations which favour high luminosity with short periods generally support injection into the population (Narayan 1987). Recent work 
by Bates et al. (2014) find $\alpha \simeq-1.4$ and $\beta \simeq 0.5$ which is very similar to the relationship found for the gamma-ray pulsar population (Perera et al. 2013). This combination of parameters leads inexorably to the conclusion that the radio luminosity decays significantly with time. Expressions which describe these trends are provided by Bates et al. (2014) and make predictions for large-scale surveys, such as the SKA.

- Death-lines and valleys. The dearth of pulsars in the lower right-hand side of the $P-\dot{P}$ diagram has long been associated with a physical limit on radio emission; for a review see Chen \& Ruderman (1993). As such, death-lines (or valleys) are frequently included in pulsar population syntheses. Very recently, a reasonable reproduction of the $P-\dot{P}$ diagram has been carried out without the adoption of a death-line, or any luminosity law (Szary et al. 2014). Instead, the study invoked an inverse correlation between radio efficiency and age. This approach appears to argue against any dependence of radio luminosity with $P$ and $\dot{P}$.

- Braking indices. Additional measurements of the braking indices of pulsars (Livingstone et al. 2007) would help to pin down the directionality in the flow of pulsars in the diagram. Indeed, some of the braking indices measured so far for young pulsars are not consistent with the locations of older pulsars and hint at the idea that some radio pulsars may evolve into magnetars (Espinoza et al. 2011). As noted by earlier authors (e.g. Tauris \& Konar 2001), a change in the braking index is required throughout the lifetime of a pulsar. This implication has yet to be fully addressed by population studies.

It is evident from the preceding discussion that many uncertainties remain in our understanding of the $P-\dot{P}$ diagram. The larger sample of pulsars provided by SKA surveys will undoubtedly help to clarify many of these issues, including the shape and the faint-end of the pulsar luminosity function. While many authors (e.g. Lyne et al. 1985; Lorimer et al. 1993) adopt power-law models of pulsars luminosities, recent work (Faucher-Giguère \& Kaspi 2006; Ridley \& Lorimer 2010; Bates et al. 2014) suggests that the parent population is more log-normal in nature.

\section{Millisecond and Binary Pulsar Surveys with SKA}

The large total collecting area, wide range of frequencies, and rapid survey speed available with SKA1 will revolutionize our understanding of the MSP population in the Milky Way. Currently, there are $\sim 300$ MSPs (defined here as $P<30 \mathrm{~ms}$ ) known in the Galaxy, including those associated with globular clusters (see the chapter by Hessels et al. 2015). Their distribution on the sky and in the Galaxy is shown in Figure 3. The primary SKA project that will drive our understanding of the MSP population is the SKA1-MID pulsar survey, which will cover 36,000 sq. deg. of sky. This is only possible with the large collecting area and very fast survey speed of SKA1MID. Surveys at high latitude may reveal new bright, stable pulsars that will contribute to pulsar timing array projects, and will fill out our understanding of the low end of the MSP luminosity function $\left(<0.1 \mathrm{mJy} \mathrm{kpc}^{2}\right.$ at $\left.1400 \mathrm{MHz}\right)$. In the plane SKA will explore a larger volume of the Galaxy than ever before to find rare systems and constrain the Galactic population of MSPs (see Section 9). The SKA1-MID pulsar survey, as defined in the SKA1 System Baseline Design is of similar sensitivity to the ongoing PALFA survey being done with the Arecibo Telescope, with flux 

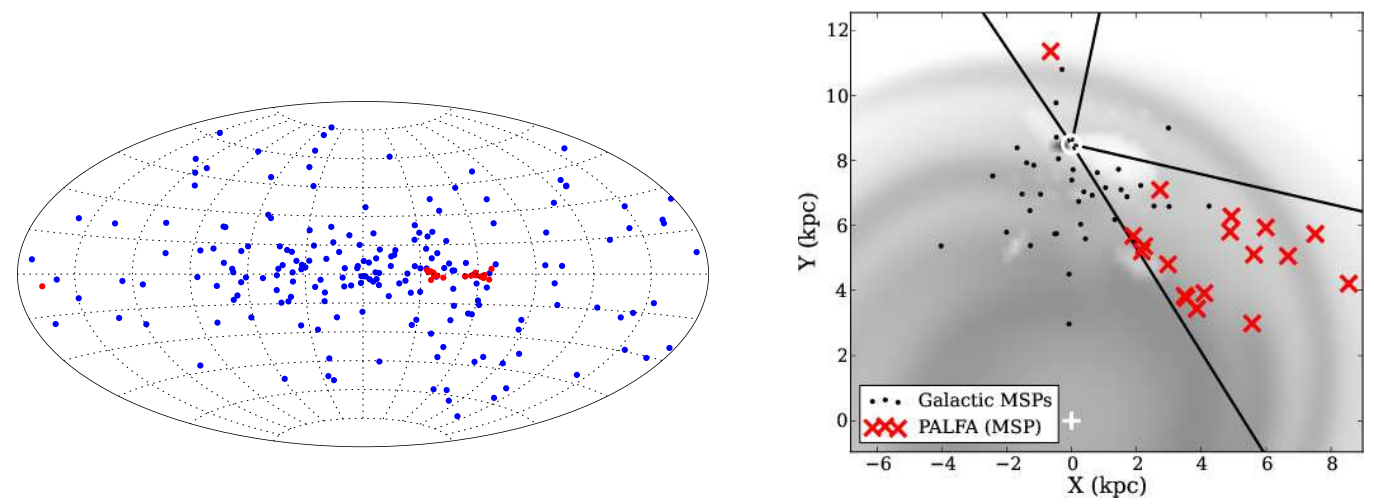

Figure 3: Left: Locations of the 216 known MSPs outside of globular clusters. Discoveries from the PALFA survey at Arecibo are shown in red. Right: Top down view of the galaxy with MSP locations marked. Again, red symbols denote PALFA MSPs (reproduced from Lazarus 2013), which are systematically more distant than the previously known population. The solid lines show the portion of the plane visible from Arecibo. SKA1 will expand that to cover nearly the entire Galaxy.

limits in the neighborhood of $40 \mu \mathrm{Jy}$. The big difference is that the SKA1-MID survey extends that sensitivity over the full visible sky, while PALFA is constrained to narrow strips of the Galactic plane visible from Arecibo (see Figure 3).

These very deep surveys are sensitive to pulsars at large dispersion measure, because of the relatively high observing frequencies, and thus probe a much larger volume of the Galaxy than previous large-area surveys. For directions in the plane, the volume sampled increases as the square of the distance probed. Figure 3 shows that PALFA is seeing a much more distant population than other surveys. SKA1-MID will expand this over nearly all of the plane, particularly towards the Galactic Bulge, where large numbers of low-mass X-ray binaries (the progenitor systems for MSPs) are known to reside. This will help constrain the total number of MSPs in the Galaxy and their density as a function of galactocentric radius, which is important for population modeling.

From an evolutionary point of view, many of the most interesting pulsars are found in tight relativistic orbits, allowing the measurement of post-Keplerian parameters. This implies that the pulsar will be accelerated for most of its orbit, requiring either short integration time with high sensitivity or sophisticated acceleration processing. Compensating for a sensitivity loss of about $10 \%$ (with respect to the baseline design) with increased observing time results in a doubling of the processing requirements to find the same relativistic binary pulsars. Hence, conducting pulsar searches with SKA1-LOW and its exceptional collecting area is a promising method for finding binary pulsars away from the Galactic plane. Indeed, our simulations show that a combined SKA1MID and SKA1-LOW survey targeting regions on and off the Galactic plane respectively finds approximately $20 \%$ more pulsars than can be found with SKA1-MID alone (Kramer \& Stappers 2014; Keane et al. 2015). Binary pulsar masses (Section 11) can only be determined if regular timing observations can be done. Though some sources may be bright enough to follow-up with other radio telescopes, it is likely that most sources will require the SKA. Sub-arraying can help alleviate the observing load for sources that are easily detectable with a fraction of the collecting area. It may also be advantageous in some cases to run pulsar timing experiments in parallel with imaging observations to boost the observing efficiency. 
When SKA2 is realized, the additional sensitivity will enable considerably more breakthrough science in this field. SKA will not only provide further sources to characterize the MSP and binary pulsar population (in particular via a high frequency survey in the Galactic bulge and central region), also the detection of giant pulses in extra-galactic MSPs (e.g. in M31 and IC 10) may be realistic.

\section{Pulsar Recycling and Formation of Exotic Binaries and Triples}

Radio pulsars have been discovered in binary systems with a variety of companions: white dwarfs (WDs), NSs, main sequence stars, and even planets. The vast majority of these systems contain an MSP with a helium WD companion. Based on stellar evolution theory, it is expected that pulsars could also be found with a helium star or a black hole companion. These systems have not yet been discovered but it is likely that the SKA will reveal such pulsars. There is a growing number of systems where the companion star is being ablated by the pulsar wind, the so-called black widows and redbacks (Roberts 2013). This is evidenced by the radio signal from the pulsar being eclipsed for some fraction of the orbit (Fruchter et al. 1988; Stappers et al. 1996; Archibald et al. 2009). These companions are low-mass semi-degenerate stars with a mass between $0.02-0.3 M_{\odot}$ (Roberts 2013; Breton et al. 2013). Whereas some studies clearly indicate that the two populations of black widows and redbacks are distinct (Chen et al. 2013) others argue in favour of a continuous evolutionary link (Benvenuto et al. 2014). This question is important to settle; as well as the long standing issue about whether they are the progenitors of the isolated MSPs (Ruderman et al. 1989; Possenti 2013; Deloye \& Bildsten 2003).

In recent years, a few binary pulsars with peculiar properties have been discovered and which indicate a hierarchical triple system origin — e.g. PSR J1903+0327 (Champion et al. 2008; Freire et al. 2011; Portegies Zwart et al. 2011). In 2013, two puzzling MSPs were discovered in eccentric binaries: PSR J2234+06 (Deneva et al. 2013) and PSR J1946+3417 (Barr et al. 2013). These two systems might also have a triple origin. However, their eccentricities and orbital periods have led to the suggestion of an alternative hypothesis of direct MSP formation via a rotationally delayed accretion-induced collapse of a massive WD (Freire \& Tauris 2014). Whereas the origin of these systems remains unclear, a triple system MSP with two WD companions (PSR J0337+1715) was announced earlier this year by Ransom et al. (2014). This amazing system must have survived at least 3 phases of mass transfer and one SN explosion and challenges current knowledge of multiple stellar system evolution (Tauris \& van den Heuvel 2014). To understand the underlying physics it is therefore important to find more of these exotic systems.

Surveys with SKA1-MID (including a coverage of the Magellanic Clouds) will indeed find many more rare systems. It is likely that SKA1 will increase the population of known pulsars and MSPs by at least a factor of 5 and thus greatly increase the number of systems which can be used to study the evolution of pulsars that are in, or have been members of, multi-stellar systems.

MSPs obtain their rapid spin (and weak B-field) via a long phase of accretion of matter from a companion star in a low-mass X-ray binary (LMXB; Backer et al. 1982; Alpar et al. 1982; Radhakrishnan \& Srinivasan 1982; Bhattacharya \& van den Heuvel 1991; Tauris et al. 2012). This is evidenced by the high incidence of binaries found among these fast spinning pulsars (see Figure 1). This model was beautifully confirmed by the discovery of the first millisecond X-ray pulsar in the 


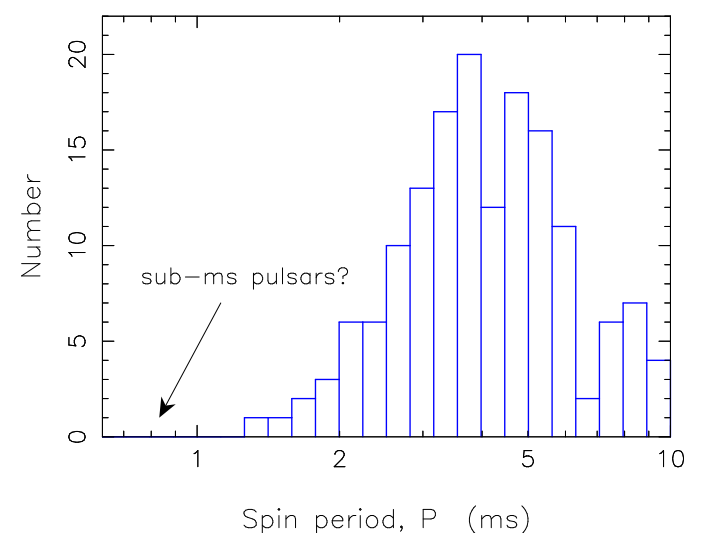

Figure 4: The observed spin period distribution of MSPs. Data taken from the ATNF Pulsar Catalogue (Manchester et al. 2005) - version 1.49, April 2014. The discovery of sub-ms MSPs with the SKA would have a huge scientific impact.

LMXB system SAX 1808.4-3658 (Wijnands \& van der Klis 1998), and more recently by the detection of the so-called transitional MSPs which undergo changes between accretion and rotational powered states (Archibald et al. 2009; Papitto et al. 2013). Many details of the recycling process, however, remain unclear. Some of the most important issues (e.g. Tauris et al. 2012, and references therein) are: the accretion-induced decay of the surface magnetic field; the maximum possible spin rate of an MSP; the Roche-lobe decoupling phase; the accretion torque reversals and the spin-up line; and the progenitors of the isolated MSPs.

There is empirical evidence that the surface magnetic field strength of NSs decays as a consequence of accretion, but the exact reason for this process is not well understood (Bhattacharya 2002). Nor is it known what dictates the fastest possible spin rate of a radio MSP. In Figure 4 we have shown the spin period distribution of radio MSPs. Does the equation-of-state (EoS) for dense nuclear matter allow for the existence of sub-ms pulsars? Or is the current spin frequency limit, slightly above $700 \mathrm{~Hz}$ (Hessels et al. 2006), set by the onset of gravitational wave (GW) emission during accretion (Chakrabarty et al. 2003), or subsequent r-mode instabilities? Alternatively, the spin-up torque might saturate due to magnetosphere-disc conditions, thus preventing sub-ms MSPs to form. In addition, it has been demonstrated (Tauris 2012) that during the final stage of mass transfer, MSPs may lose up to 50\% of their rotational energy when the donor star decouples from its Roche lobe.

\subsection{The contribution of SKA}

As described in detail elsewhere in this book, the large scale surveys performed with SKA1 will lead to a significant increase in the number of catalogued MSPs. That will also include several new exotic (and therefore intrinsically rare, possibly unprecedented) objects, suitable to directly address some of the key-questions reported above: i.e. the discovery of even a single MSP spinning at a period well below $1 \mathrm{~ms}$ would provide strong constraints on the NS structure and the EoS of dense nuclear matter. In the context of evolutionary studies, it will be particularly interesting to use SKA1-MID for performing a deep search for pulsars at a higher frequency (between 2 and $3 \mathrm{GHz}$ ) 
than ever for an all-sky survey. In fact, at those frequencies, the absorbing/eclipsing effects of the plasma released by the companion star are significantly reduced with respect to what happens at the lower frequencies typically used in all the past large scale surveys. That could finally unveil the radio signal from several ultra close-orbit binary pulsars in the last phases of their recycling, i.e. the transitional MSPs which are among the most precious systems for investigating the still unanswered issues discussed in this section.

SKA2 will then provide an extremely large population of recycled pulsars, eventually enabling a statistically sound comparison between the properties of the various classes of binary and isolated MSPs, thus firmly establishing relationships and evolutionary connections. Similarly, it has been shown in several works (e.g. Possenti et al. 1999; Tauris et al. 2012, and references therein) that the availability of a large population of MSPs (maybe including still unknown MSPs with surface B-fields below $10^{7} \mathrm{G}$, or MSPs with relatively high B-fields) would constrain the physical processes during the last stages of accretion. This includes new knowledge on the evolutionary phases experienced by a pulsar close to the spin-up line and/or the occurrence of GW emission from the accreting NS, as well as the radio emission mechanism in old NSs (i.e. the death-line for low B-field pulsars).

\section{Double Neutron Star and Neutron Star/Black Hole Systems}

In 1974 a new class of radio pulsars was discovered with the detection of the first double NS system (Hulse \& Taylor 1975). Today we know a dozen double NS systems. The formation and evolution of such relativistic binaries (tight systems with massive compact stars) is a key topic in modern astrophysics with many applications to fundamental physics in general. In particular, double NS systems are important for: pre-SN binary evolution and explosion physics, measurements of NS masses, testing theories of gravity, and their mergers are prime candidates for detection of high-frequency gravitational waves with LIGO/VIRGO within the next few years.

Double NS binaries are important for both stellar astrophysics and SN explosion modelling since their observed orbital characteristics represent a fossil relic of the last (second) SN explosion. Hence, from observations of double NS binaries we can place constraints on both the pre-SN conditions and the explosion mechanism itself. Many of the systems have rather small eccentricities and small masses of the last (second) formed NS. This is indeed expected if these SNe were either caused by electron capture SNe (Podsiadlowski et al. 2004) and/or a result of pre-SN ultrastripping of the collapsing star (Tauris et al. 2013a). However, also higher birth masses of NSs are expected from stellar evolution (Tauris et al. 2011) and the many new double NS binaries anticipated to be discovered by the SKA will finally probe the full distribution of NS birth masses in these systems (see Section 11). The SKA is very likely to discover more double pulsar systems like PSR J0737-3039A/B. From observations (Breton et al. 2008, 2012) and modelling (Lyutikov $\&$ Thompson 2005) of interactions, via eclipse light curves, between the emission beam of one pulsar with the magnetosphere of its companion pulsar, detailed knowledge can be obtained with respect to the magnetospheric structure (see also Karastergiou et al. 2015), the plasma properties, and relativistic precession of pulsars.

With the many new pulsar discoveries to be expected with the SKA there is realistic hope that they will also include a few long-sought-after NS-black hole (NS-BH) binaries. NS-BH binaries 
have the potential to revolutionize gravity tests (Kramer et al. 2006b; Wex 2014; Shao et al. 2015) and will also bring new insight to the final stages of massive stellar evolution and SNe.

Understanding the NS-NS and NS-BH populations is important since their merger events are prime candidate sources for the upcoming detection of high-frequency gravitational waves with LIGO/VIRGO. The event rate of detections is expected to be of the order 0.1-10 per week (Voss \& Tauris 2003; Abadie et al. 2010) and this rate should match the observed distributions of orbital separations and eccentricities among Galactic NS-NS and NS-BH sources. The LIGO/VIRGO detection rate is based on the Galactic merger rate of NS-NS and NS-BH systems which is of the order a few $\mathrm{Myr}^{-1}$. However, this number is highly uncertain due to the many complex factors involved in computing the formation rate of these systems (e.g. Voss \& Tauris 2003).

\section{Neutron Star Masses}

The masses of NSs play a vital role in a number of aspects pertaining to their structure, formation and evolution. So far, all pulsar mass measurements rely on one form or another of dynamical proxies offered by binary systems. The starting point is pulsar timing, which provides a very accurate determination of the mass function of the system and links the orbital inclination to the two masses. Supplementing it with two additional constraints therefore breaks the degeneracy. This can be achieved in various ways: i) Relativistic binaries - usually double NS systems - allow for the measurement of post-Keplerian parameters (such as the Shapiro delay and the periastron advance) that each yield an extra constraint, see Shao et al. (2015); ii) Binaries containing an optically bright companion may provide its projected radial velocity via spectroscopy, a companion mass determination (e.g. via the atmosphere modelling of WDs, van Kerkwijk et al. 2005), or a measure of the mass ratio and orbital inclination via light curve modelling (e.g. Breton et al. 2013).

Since any given EoS (see e.g. the chapter by Watts et al. 2015) sets a maximum mass stability limit, hunting for massive pulsars can provide an efficient way of constraining its nature. With a significant sample of NS masses, however, one can statistically constrain their EoS. Current observational evidence (Demorest et al. 2010; Antoniadis et al. 2013) disfavours a "soft" EoS, which cannot reach much beyond the measured $2 M_{\odot}$.

Pulsar masses are also fundamental tracers of their formation and subsequent evolution via accretion. The birth mass of a NS relates to its formation and reflects the SN physics (Tauris et al. 2011; Ferdman et al. 2013, 2014). Hence the presence of multiple modes in the birth mass distribution will probe their formation channels (i.e. iron core-collapse $\mathrm{SNe}$ and electron capture $\mathrm{SNe}$ ). The mass distribution of NS-NS and NS-WD binaries was recently analysed (Kiziltan et al. 2013) and it was concluded that they peak at $1.33 M_{\odot}$ and $1.55 M_{\odot}$, respectively, with the first having a nearly symmetrical spread of $0.12 M_{\odot}(1-\sigma)$ while the other one being skewed towards higher masses. This arises from the fact that the observed pulsar masses are convoluted by the evolutionary history of the binary system in which they lie. For instance, pulsars with low-mass WD companions have gone through a long-lasting LMXB phase which has likely increased their mass anywhere from $\sim 0.1-0.5 \mathrm{M}_{\odot}$ (see the previous Section 9 on pulsar recycling). Double NS binaries, on the other hand, should reflect the birth mass more closely since the first-born NS only accretes $<0.01 M_{\odot}$ during/following a common-envelope evolution (Tauris et al. 2012) while the 
second NS should have accreted none. Comparing the masses from the two sub-populations might therefore provide a powerful probe for mass transfer in binaries.

\subsection{The contribution of SKA}

SKA1 is expected to increase the number of known pulsar binaries by more than a factor of five, which should therefore grow the NS mass sample size by a similar factor. This naive assumption is certainly justified for relativistic NS-NS binaries since their mass measurements rely on radio timing only, while in other binaries they suffer the caveat that they require optical data as well. Projects such as the Thirty-Meter Telescope and the European Extremely Large Telescope will provide a ten-fold increase in sensitivity, thus enabling to push down the luminosity function of the probed systems to sustain the growth of mass measurements in these systems as well.

Given a factor of ten increase in the mass sample with SKA2, how well can we expect to determine the minimum and maximum mass range of NSs? Based on the distribution of Kiziltan et al. (2013), i.e. a normal distribution $\mathscr{N}\left(\mu=1.33 M_{\odot}, \sigma=0.12 M_{\odot}\right)$, the probability of finding a mass below the putative $1.08 \mathrm{M}_{\odot}$ lower limit is $1.86 \%$, which implies that a 200-NS masses sample from NS-NS systems would have a $97.7 \%$ chance of containing such a low-mass NS (should they exist). If a low-mass limit cutoff exists, it will as well manifest as a skewness in the distribution, which would lack data at the low end. Regarding the upper mass limit, we turn toward NS-WD systems. Here, for simplicity, we assume that they have the same underlying birth-mass distribution as the NS-NS systems (i.e. $\mathscr{N}(\mu=1.33, \sigma=0.12)$ ), and have then accreted some arbitrary mass that we model using a gamma distribution with a scale parameter 0.15 and a shape parameter 2.0. Based on this we infer the probability of finding a mass above $2.4 \mathrm{M}_{\odot}$ is $0.42 \%$, thus corresponding to $47 \%$ odds of having a NS above that limit in a sample of 150 (should such a NS exist). From the distribution of NS masses and eccentricities it is even possible to place limits on the gravitational binding energy of NSs (Podsiadlowski et al. 2005), released via neutrinos during the SN event. Thus future SKA observations can also via this method help to narrow down the EoS of dense nuclear matter (see the chapter by Watts et al. 2015).

\section{Neutron Star Kicks and Velocities}

Soon after the discovery of pulsars, it was recognized that their average space velocity greatly exceeds that of their progenitor stars (Gunn \& Ostriker 1970). At the present time, with proper motions measured for hundreds of pulsars via timing or VLBI, it is clear that most radio pulsars receive a large kick $\left(\sim 400 \mathrm{~km} \mathrm{~s}^{-1}\right)$ during their formation event (Hobbs et al. 2005). However, a number of plausible processes remain for the exact physical mechanism responsible for imparting this kick during the SN (Lai et al. 2001; Janka 2012). Many pulsars are observed in globular clusters which have small escape velocities of $\lesssim 40 \mathrm{~km} \mathrm{~s}^{-1}$. Theoretical studies of electron capture $\mathrm{SNe}$ and the accretion-induced collapse (AIC) of massive WDs provide a plausible NS formation mechanism which predicts a low kick velocity (Podsiadlowski et al. 2004; Schwab et al. 2010; Tauris et al. 2013b). In addition, there is the interesting question of a possible alignment at birth between the pulsar velocity vector and its spin axis, as suggested by recent observational evidence and modelling (Noutsos et al. 2013). 
In order to differentiate between the competing models for NS birth kicks, reliable velocity estimates are needed for a larger fraction of the known pulsar population. The fact that the radial component of velocity is inaccessible for pulsars in all but a very few cases sharpens the need for a large sample of objects. As of early 2014, transverse velocity estimates are available for around 270 pulsars $^{5}$, but most of these are of low precision, either because of the proper motion measurement itself or (more commonly) uncertainties in the pulsar distance provided from the pulsar dispersion measure and the NE2001 model of the Galactic electron density distribution (Cordes \& Lazio 2002; Deller et al. 2009, 2011). In order to improve the reliability and size of the pulsar velocity distribution sample, three things are needed: i) Additional proper motion measurements, via timing or high resolution astrometry; ii) Accurate, model-independent distance measurements for a larger fraction of the objects with proper motion measurements (again via timing and - more commonly - high resolution astrometry); and iii) A better model of the Galactic electron density distribution, allowing more reliable model-dependent distance estimates for the remaining pulsars.

\subsection{The contribution of SKA}

With its phenomenal sensitivity, SKA will be able to measure proper motions and parallax distances for radio pulsars via the current approaches (high precision timing and high resolution astrometric observations) but with greatly improved accuracy, and for a much larger number of systems (Smits et al. 2011). For recycled pulsars with very stable rotation properties, SKA will be able to measure proper motion and often parallax via timing. In many cases, this will come as a byproduct of programs undertaken for other purposes, as described earlier in this chapter. The remaining pulsars can be addressed via astrometry. With the moderate baselines of SKA1-MID $(\sim 100 \mathrm{~km})$ it will be possible to measure positions accurate to several milli-arcseconds in a single observation for all but the faintest pulsars. This will be sufficient to measure a proper motion for the majority of pulsars if several observations are made over a multi-year period.

However, to obtain the parallax measurements necessary to anchor the distance scale, much higher angular resolution will be needed. This can be achieved using a phased SKA1-MID and SKA1-SUR as part of a wider intercontinental VLBI network.

The biggest impact of SKA2 for pulsar velocity studies is the extension of baselines to SKA1MID. With a resolution of a few tens of milli-arcseconds or better at $\mathrm{GHz}$ frequencies, coupled with $10 \times$ higher sensitivity than SKA1-MID, SKA2 will be able to perform ultra-precision astrometry on the majority of the entire radio pulsar population visible from the site. In terms of providing the accurate velocity and distance information needed to characterise the pulsar population, SKA2 as currently envisaged encompasses all of the required capabilities.

\section{A brief summary of the impact of early phase SKA1 (50\% SKA1)}

We end this chapter with a brief summary of the outlooks for early deployment of SKA1 and its expected impact on pulsar science (see also Kramer \& Stappers 2014).

Early deployment of SKA1 at 50\% sensitivity would not provide access to the faintest pulsars of the population. Simulations for SKA1 by Keane et al. (2015) predict detection of $\sim 10000$ normal pulsars and $~ 1500$ MSPs, whereas for early phase SKA1 (50\% SKA1) the expected numbers

\footnotetext{
${ }^{5}$ http://www.atnf.csiro.au/people/pulsar/psrcat/
} 
are roughly 6000 and 750, respectively (keeping the integration time the same). To partly compensate for the sensitivity loss one could, for example, double the integration time. In that case the predicted number of detections are about 7500 normal pulsars and 950 MSPs. However, an increase in integration time would also largely increase the required amount of data processing given that the latter scales with the integration time cubed. An additional handicap for discovering the fast spinning MSPs (as well as double NS and NS-BH binaries) would be the increase in Dopplersmearing of the radio pulses using longer integration times, given that many MSPs are being highly accelerated in tight binaries.

The expected sensitivity limit for the SKA1-MID survey can be compared with, for example, the HTRU survey (Keith et al. 2010) ongoing at the Parkes Radio Telescope and the Arecibo PALFA survey (Cordes et al. 2006). Whereas the sensitivity for SKA1 50\% would only be about half the sensitivity of the Arecibo PALFA survey (which, on the other hand, has a very limited sky coverage), it would still be significantly better than the HTRU survey by a factor of 2-6, depending on the Galactic latitude. Another comparison can be made to the complete 64 dishes MeerKAT Radio Telescope. MeerKAT will be operational in 2017 and will later become an integrated part of SKA1-MID. Early deployment of SKA1 at 50\% would still have a sensitivity about two times better than that of MeerKAT.

For the measurement of NS kicks and velocities, early-phase observations with SKA1 at 50\% sensitivity would be unable to access the faintest pulsars, limiting the maximum number of accurate transverse velocity measurements that could be made (whether by timing with SKA1-MID, imaging with SKA1-MID, or imaging with SKA1-VLBI). The pool of remaining targets still represents a factor-of-many increase over the current state of affairs, and in any case is likely larger than the observing time available in early-phase SKA1.

As pointed out elsewhere, the SKA1 is not only a simple stepping stone towards SKA2. Due to limitations in processing power, it is unlikely that the full area of the completed SKA can be utilized for a blind, large-scale survey for some time to come. SKA1 with a highly concentrated core represents a significant fraction of collecting area usable for surveys with SKA2. Although the outcome will depend on the number of dishes, the bandwidth and the central frequency of a given survey (or a composite survey covered by SKA1-LOW and SKA1-MID), the total number of beams, and the computer power available, significant achievements can even be made in finding MSPs and binary pulsars during early deployment pulsar searching at the 50\% SKA level.

\section{References}

Abadie, J., Abbott, B. P., Abbott, R., et al. 2010, Classical and Quantum Gravity, 27, 173001

Aguilera, D. N., Pons, J. A., \& Miralles, J. A. 2008, A\&A, 486, 255

Alpar, M. A., Cheng, A. F., Ruderman, M. A., \& Shaham, J. 1982, Nature, 300, 728

Anderson, G. E., Gaensler, B. M., Slane, P. O., et al. 2012, ApJ, 751, 53

Antoniadis, J., Freire, P. C. C., Wex, N., et al. 2013, Science, 340, 448

Archibald, A. M., Stairs, I. H., Ransom, S. M., et al. 2009, Science, 324, 1411

Backer, D. C. 1970, Nature, 228, 42

Backer, D. C., Kulkarni, S. R., Heiles, C., Davis, M. M., \& Goss, W. M. 1982, Nature, 300, 615

Baring, M. G., \& Harding, A. K. 2001, ApJ, 547, 929 
Barr, E. D., Champion, D. J., Kramer, M., et al. 2013, MNRAS, 435, 2234

Bates, S. D., Lorimer, D. R., Rane, A., \& Swiggum, J. 2014, MNRAS, 439, 2893

Bates, S. D., Johnston, S., Lorimer, D. R., et al. 2011, MNRAS, 411, 1575

Beloborodov, A. M., \& Thompson, C. 2007, ApJ, 657, 967

Benvenuto, O. G., De Vito, M. A., \& Horvath, J. E. 2014, ApJ, 786, L7

Bhattacharya, D. 2002, Journal of Astrophysics and Astronomy, 23, 67

Bhattacharya, D., \& van den Heuvel, E. P. J. 1991, Physics Reports, 203, 1

Bhattacharya, D., Wijers, R. A. M. J., Hartman, J. W., \& Verbunt, F. 1992, A\&A, 254, 198

Bogdanov, S., Ng, C.-Y., \& Kaspi, V. M. 2014, ApJ, 792, L36

Bower, G. C., Deller, A., Demorest, P., et al. 2014, ApJ, 780, L2

Breton, R. P., Kaspi, V. M., Kramer, M., et al. 2008, Science, 321, 104

Breton, R. P., Kaspi, V. M., McLaughlin, M. A., et al. 2012, ApJ, 747, 89

Breton, R. P., van Kerkwijk, M. H., Roberts, M. S. E., et al. 2013, ApJ, 769, 108

Burgay, M., Rea, N., Israel, G. L., et al. 2006, MNRAS, 372, 410

Camilo, F., Ransom, S. M., Chatterjee, S., Johnston, S., \& Demorest, P. 2012, ApJ, 746, 63

Camilo, F., Ransom, S. M., Halpern, J. P., \& Reynolds, J. 2007a, ApJ, 666, L93

Camilo, F., Ransom, S. M., Halpern, J. P., et al. 2006, Nature, 442, 892

Camilo, F., Reynolds, J., Johnston, S., et al. 2007b, ApJ, 659, L37

Camilo, F., Cognard, I., Ransom, S. M., et al. 2007c, ApJ, 663, 497

Chakrabarty, D., Morgan, E. H., Muno, M. P., et al. 2003, Nature, 424, 42

Champion, D. J., Ransom, S. M., Lazarus, P., \& et al. 2008, Science, 320, 1309

Chen, H.-L., Chen, X., Tauris, T. M., \& Han, Z. 2013, ApJ, 775, 27

Chen, K., \& Ruderman, M. 1993, ApJ, 402, 264

Chennamangalam, J., \& Lorimer, D. R. 2014, MNRAS, 440, L86

Contopoulos, I., Kazanas, D., \& Fendt, C. 1999, ApJ, 511, 351

Contopoulos, I., \& Spitkovsky, A. 2006, ApJ, 643, 1139

Cordes, J. M., \& Lazio, T. J. W. 2002, ArXiv: astro-ph/0207156, astro-ph/0207156

Cordes, J. M., Freire, P. C. C., Lorimer, D. R., et al. 2006, ApJ, 637, 446

Crawford, F., Hessels, J. W. T., \& Kaspi, V. M. 2007, ApJ, 662, 1183

Damour, T., \& Taylor, J. H. 1992, Phys. Rev. D, 45, 1840

de Luca, A. 2008, in American Institute of Physics Conference Series, Vol. 983, 40 Years of Pulsars: Millisecond Pulsars, Magnetars and More, ed. C. Bassa, Z. Wang, A. Cumming, \& V. M. Kaspi, 311-319

Deller, A. T., Tingay, S. J., Bailes, M., \& Reynolds, J. E. 2009, ApJ, 701, 1243

Deller, A. T., Brisken, W. F., Chatterjee, S., et al. 2011, in 20th Meeting of the European VLBI Group for Geodesy and Astronomy, held in Bonn, Germany, March 29-30, 2011, Eds: W. Alef, S. Bernhart, and A. Nothnagel, Institut für Geodäsie und Geoinformation, Rheinischen Friedrich-Wilhelms-Universität Bonn, p. 178-182, ed. W. Alef, S. Bernhart, \& A. Nothnagel, 178-182

Deloye, C. J., \& Bildsten, L. 2003, ApJ, 598, 1217

Demorest, P. B., Pennucci, T., Ransom, S. M., Roberts, M. S. E., \& Hessels, J. W. T. 2010, Nature, 467, 1081

Deneva, J. S., Stovall, K., McLaughlin, M. A., et al. 2013, ApJ, 775, 51 
Dib, R., \& Kaspi, V. M. 2014, ApJ, 784, 37

Dib, R., Kaspi, V. M., \& Gavriil, F. P. 2008, ApJ, 673, 1044

Eatough, R. P., Lazio, T. J. W., Casanellas, J., \& et al., 2015, "Observing Radio Pulsars in the Galactic Centre with the Square Kilometre Array", in proc. Advancing Astrophysics with the Square Kilometre Array, PoS(AASKA14)045

Eatough, R. P., Falcke, H., Karuppusamy, R., et al. 2013, Nature, 501, 391

Emmering, R. T., \& Chevalier, R. A. 1989, ApJ, 345, 931

Esamdin, A., Lyne, A. G., Graham-Smith, F., et al. 2005, MNRAS, 356, 59

Espinoza, C. M., Lyne, A. G., Kramer, M., Manchester, R. N., \& Kaspi, V. M. 2011, ApJ, 741, L13

Faucher-Giguère, C.-A., \& Kaspi, V. M. 2006, ApJ, 643, 332

Ferdman, R. D., Stairs, I. H., Kramer, M., et al. 2013, ApJ, 767, 85

-. 2014, MNRAS, 443, 2183

Freire, P. C. C., \& Tauris, T. M. 2014, MNRAS, 438, L86

Freire, P. C. C., Bassa, C. G., Wex, N., et al. 2011, MNRAS, 412, 2763

Fruchter, A. S., Stinebring, D. R., \& Taylor, J. H. 1988, Nature, 333, 237

Gaensler, B. M., Bock, D. C.-J., \& Stappers, B. W. 2000, ApJ, 537, L35

Gavriil, F. P., Gonzalez, M. E., Gotthelf, E. V., et al. 2008, Science, 319, 1802

Goldreich, P., \& Julian, W. H. 1969, ApJ, 157, 869

Gotthelf, E. V., Halpern, J. P., \& Alford, J. 2013, ApJ, 765, 58

Guendelman, E. I., \& Chelouche, D. 2011, International Journal of Modern Physics E, 20, 100

Gunn, J. E., \& Ostriker, J. P. 1970, ApJ, 160, 979

Halpern, J. P., \& Gotthelf, E. V. 2010, ApJ, 709, 436

Hessels, J. W. T., Possenti, A., Bailes, M., \& et al., 2015, "Pulsars in Globular Clusters with the SKA", in proc. Advancing Astrophysics with the Square Kilometre Array, PoS(AASKA14)047

Hessels, J. W. T., Ransom, S. M., Stairs, I. H., et al. 2006, Science, 311, 1901

Hobbs, G., Lorimer, D. R., Lyne, A. G., \& Kramer, M. 2005, MNRAS, 360, 974

Hulse, R. A., \& Taylor, J. H. 1975, ApJ, 195, L51

Janka, H.-T. 2012, Annual Review of Nuclear and Particle Science, 62, 407

Kaplan, D. L., Gaensler, B. M., Kulkarni, S. R., \& Slane, P. O. 2006, ApJS, 163, 344

Karastergiou, A., Johnston, S., Andersson, N., \& et al. 2015, "Understanding pulsar magnetospheres with the SKA", in proc. Advancing Astrophysics with the Square Kilometre Array, PoS(AASKA14)038

Kaspi, V. M. 2010, Proceedings of the National Academy of Science, 107, 7147

Kaspi, V. M., \& McLaughlin, M. A. 2005, ApJ, 618, L41

Kaspi, V. M., Archibald, R. F., Bhalerao, V., et al. 2014, ApJ, 786, 84

Keane, E. F., Bhattacharyya, B., Kramer, M., \& et al. 2015, "A Cosmic Census of Radio Pulsars with the SKA", in proc. Advancing Astrophysics with the Square Kilometre Array, PoS(AASKA14)040

Keane, E. F., \& Kramer, M. 2008, MNRAS, 391, 2009

Keane, E. F., Ludovici, D. A., Eatough, R. P., et al. 2010, MNRAS, 401, 1057

Keane, E. F., \& McLaughlin, M. A. 2011, Bulletin of the Astronomical Society of India, 39, 333

Keith, M. J., Shannon, R. M., \& Johnston, S. 2013, MNRAS, 432, 3080

Keith, M. J., Jameson, A., van Straten, W., et al. 2010, MNRAS, 409, 619 
Kiziltan, B., Kottas, A., De Yoreo, M., \& Thorsett, S. E. 2013, ApJ, 778, 66

Klus, H., Ho, W. C. G., Coe, M. J., Corbet, R. H. D., \& Townsend, L. J. 2014, MNRAS, 437, 3863

Kramer, M., Lyne, A. G., O’Brien, J. T., Jordan, C. A., \& Lorimer, D. R. 2006a, Science, 312, 549

Kramer, M., \& Stappers, B. W. 2014, SKA Pulsar Science Assessment Workshop report (available at https://indico.skatelescope.org/conferenceDisplay.py?confId=260)

Kramer, M., Stappers, B. W., Jessner, A., Lyne, A. G., \& Jordan, C. A. 2007, MNRAS, 377, 107

Kramer, M., Stairs, I. H., Manchester, R. N., et al. 2006b, Science, 314, 97

Lai, D., Chernoff, D. F., \& Cordes, J. M. 2001, ApJ, 549, 1111

Lazarus, P. 2013, in IAU Symposium, Vol. 291, IAU Symposium, ed. J. van Leeuwen, 35-40

Lazarus, P., Kaspi, V. M., Champion, D. J., Hessels, J. W. T., \& Dib, R. 2012, ApJ, 744, 97

Lee, K. J., Guillemot, L., Yue, Y. L., Kramer, M., \& Champion, D. J. 2012, MNRAS, 424, 2832

Levin, L., Bailes, M., Bates, S., et al. 2010, ApJ, 721, L33

Li, J., Spitkovsky, A., \& Tchekhovskoy, A. 2012, ApJ, 746, 60

Liu, K., Wex, N., Kramer, M., Cordes, J. M., \& Lazio, T. J. W. 2012, ApJ, 747, 1

Livingstone, M. A., Kaspi, V. M., Gavriil, F. P., et al. 2007, Ap\&SS, 308, 317

Lorimer, D. R., Bailes, M., Dewey, R. J., \& Harrison, P. A. 1993, MNRAS, 263, 403

Lorimer, D. R., \& Kramer, M. 2004, Handbook of Pulsar Astronomy, Cambridge University Press

Lorimer, D. R., Lyne, A. G., McLaughlin, M. A., et al. 2012, ApJ, 758, 141

Lynch, R. S., \& Kaspi, V. M. 2015, in preparation

Lyne, A., Hobbs, G., Kramer, M., Stairs, I., \& Stappers, B. 2010, Science, 329, 408

Lyne, A. G., Manchester, R. N., \& Taylor, J. H. 1985, MNRAS, 213, 613

Lyutikov, M. 2002, ApJ, 580, L65

Lyutikov, M., \& Thompson, C. 2005, ApJ, 634, 1223

Manchester, R. N., Hobbs, G. B., Teoh, A., \& Hobbs, M. 2005, AJ, 129, 1993

McLaughlin, M. A., Lyne, A. G., Lorimer, D. R., et al. 2006, Nature, 439, 817

Mori, K., Gotthelf, E. V., Zhang, S., et al. 2013, ApJ, 770, L23

Narayan, R. 1987, ApJ, 319, 162

Narayan, R., \& Ostriker, J. P. 1990, ApJ, 352, 222

Noutsos, A., Schnitzeler, D. H. F. M., Keane, E. F., Kramer, M., \& Johnston, S. 2013, MNRAS, 430, 2281

Olausen, S. A., \& Kaspi, V. M. 2014, ApJS, 212, 6

Olausen, S. A., Zhu, W. W., Vogel, J. K., et al. 2013, ApJ, 764, 1

Papitto, A., Ferrigno, C., Bozzo, E., et al. 2013, Nature, 501, 517

Pavlov, G. G., Sanwal, D., Garmire, G. P., \& Zavlin, V. E. 2002, in Astronomical Society of the Pacific Conference Series, Vol. 271, Neutron Stars in Supernova Remnants, ed. P. O. Slane \& B. M. Gaensler, 247

Perera, B. B. P., McLaughlin, M. A., Cordes, J. M., et al. 2013, ApJ, 776, 61

Perna, R., Viganò, D., Pons, J. A., \& Rea, N. 2013, MNRAS, 434, 2362

Pfahl, E., \& Loeb, A. 2004, ApJ, 615, 253

Phinney, E. S., \& Blandford, R. D. 1981, MNRAS, 194, 137

Podsiadlowski, P., Dewi, J. D. M., Lesaffre, P., et al. 2005, MNRAS, 361, 1243

Podsiadlowski, P., Langer, N., Poelarends, A. J. T., et al. 2004, ApJ, 612, 1044

Popov, S. B., Pons, J. A., Miralles, J. A., Boldin, P. A., \& Posselt, B. 2010, MNRAS, 401, 2675 
Popov, S. B., \& Turolla, R. 2012, Ap\&SS, 341, 457

Portegies Zwart, S., van den Heuvel, E. P. J., van Leeuwen, J., \& Nelemans, G. 2011, ApJ, 734, 55

Possenti, A. 2013, in IAU Symposium, Vol. 291, IAU Symposium, ed. J. van Leeuwen, 121-126

Possenti, A., Colpi, M., Geppert, U., Burderi, L., \& D’Amico, N. 1999, ApJS, 125, 463

Radhakrishnan, V., \& Srinivasan, G. 1982, Current Science, 51, 1096

Ransom, S. M., Stairs, I. H., Archibald, A. M., et al. 2014, Nature, 505, 520

Rea, N., Israel, G. L., Pons, J. A., et al. 2013, ApJ, 770, 65

Ridley, J. P., \& Lorimer, D. R. 2010, MNRAS, 404, 1081

Roberts, M. S. E. 2013, in IAU Symposium, Vol. 291, IAU Symposium, ed. J. van Leeuwen, 127132

Ruderman, M., Shaham, J., \& Tavani, M. 1989, ApJ, 336, 507

Schwab, J., Podsiadlowski, P., \& Rappaport, S. 2010, ApJ, 719, 722

Shannon, R. M., \& Johnston, S. 2013, MNRAS, 435, L29

Shao, L., Stairs, I. H., Antoniadis, J., \& et al., 2015, "Testing Gravity with Pulsars in the SKA Era", in proc. Advancing Astrophysics with the Square Kilometre Array, PoS(AASKA14)042

Smits, R., Tingay, S. J., Wex, N., Kramer, M., \& Stappers, B. 2011, A\&A, 528, A108

Spitkovsky, A. 2006, ApJ, 648, L51

Spitler, L. G., Lee, K. J., Eatough, R. P., et al. 2014, ApJ, 780, L3

Stappers, B. W., Bailes, M., Lyne, A. G., et al. 1996, ApJ, 465, L119

Stollman, G. M. 1987, A\&A, 171, 152

Szary, A., Zhang, B., Melikidze, G. I., Gil, J., \& Xu, R.-X. 2014, ApJ, 784, 59

Tauris, T. M. 2012, Science, 335, 561

Tauris, T. M., \& Konar, S. 2001, A\&A, 376, 543

Tauris, T. M., Langer, N., \& Kramer, M. 2011, MNRAS, 416, 2130

—. 2012, MNRAS, 425, 1601

Tauris, T. M., Langer, N., Moriya, T. J., et al. 2013a, ApJ, 778, L23

Tauris, T. M., \& Manchester, R. N. 1998, MNRAS, 298, 625

Tauris, T. M., Sanyal, D., Yoon, S.-C., \& Langer, N. 2013b, A\&A, 558, A39

Tauris, T. M., \& van den Heuvel, E. P. J. 2006, Formation and evolution of compact stellar X-ray sources (Cambridge University Press), 623-665

-. 2014, ApJ, 781, L13

Thompson, C., \& Duncan, R. C. 1995, MNRAS, 275, 255

-. 1996, ApJ, 473, 322

Thompson, C., Lyutikov, M., \& Kulkarni, S. R. 2002, ApJ, 574, 332

Timokhin, A. N. 2010, MNRAS, 408, L41

Turolla, R. 2009, in Astrophysics and Space Science Library, Vol. 357, Astrophysics and Space Science Library, ed. W. Becker, 141

van Kerkwijk, M. H., Bassa, C. G., Jacoby, B. A., \& Jonker, P. G. 2005, in Astronomical Society of the Pacific Conference Series, Vol. 328, Binary Radio Pulsars, ed. F. A. Rasio \& I. H. Stairs, $357-+$

Vivekanand, M., \& Narayan, R. 1981, Journal of Astrophysics and Astronomy, 2, 315

Voss, R., \& Tauris, T. M. 2003, MNRAS, 342, 1169

Wang, N., Manchester, R. N., \& Johnston, S. 2007, MNRAS, 377, 1383 
Watts, A., Xu, R., Espinoza, C., \& et al. 2015, "Probing the neutron star interior and the Equation of State of cold dense matter with the SKA", in proc. Advancing Astrophysics with the Square Kilometre Array, PoS(AASKA14)043

Weisskopf, M. C., Swartz, D. A., Carramiñana, A., et al. 2006, ApJ, 652, 387

Weltevrede, P., \& Johnston, S. 2008, MNRAS, 387, 1755

Weltevrede, P., Johnston, S., \& Espinoza, C. M. 2011, MNRAS, 411, 1917

Wex, N. 2014, ArXiv: 1402.5594

Wijnands, R., \& van der Klis, M. 1998, Nature, 394, 344

Young, M. D. T., Chan, L. S., Burman, R. R., \& Blair, D. G. 2010, MNRAS, 402, 1317

Young, N. J., Stappers, B. W., Lyne, A. G., et al. 2013, MNRAS, 429, 2569

Young, N. J., Stappers, B. W., Weltevrede, P., Lyne, A. G., \& Kramer, M. 2012, MNRAS, 427, 114

Zhu, W. W., Kaspi, V. M., McLaughlin, M. A., et al. 2011, ApJ, 734, 44 